\title{
On the measurement of vortex filament lifetime statistics in turbulence
}

\author{
Luca Biferale, ${ }^{1}$ Andrea Scagliarini, ${ }^{1}$ and Federico Toschi ${ }^{2}$ \\ (International Collaboration for Turbulence Research) \\ ${ }^{1}$ Department of Physics and INFN, University of Tor Vergata, Via della Ricerca Scientifica 1, \\ 00133 Rome, Italy \\ ${ }^{2}$ Department of Physics and Department of Mathematics and Computer Science and J. M. Burgers Centre \\ for Fluid Dynamics, Eindhoven University of Technology, 5600 MB Eindhoven, The Netherlands
}

(Received 10 November 2009; accepted 29 April 2010; published online 4 June 2010)

\begin{abstract}
A numerical study of turbulence seeded with light particles is presented. We analyze the statistical properties of coherent, small-scale structures by looking at the trapping events of light particles inside vortex filaments. We study the properties of particles attracting set, measuring its fractal dimension and the probability that the separation between two particles remains within the dissipative scale, even for time lapses as long as the large-scale correlation time, $T_{L}$. We show how to estimate the vortex lifetime by studying the moment of inertia of bunches of particles, showing the presence of an exponential lifetime distribution, with events up to $T_{L}$. (C) 2010 American Institute of Physics. [doi:10.1063/1.3431660]
\end{abstract}

Vorticity dynamics, in general, and vortex filaments, in particular, have been the subject of many theoretical, phenomenological and experimental studies. ${ }^{1-4}$ According to their inertia properties particles respond differently to fluctuations of the advecting-Eulerian-velocity field producing locally nonhomogeneous concentration, a phenomenon dubbed as "preferential concentration." pability of strongly concentrating in high vorticity regions, very light particles (i.e., small bubbles in water) have been used to visualize small scale vortex filaments, ${ }^{7,8}$ and to measure pressure statistics. ${ }^{9}$ Similar phenomena, based on complex response of microscopic hydrogen particles in quantum fluids, have also been exploited recently to visualize quantized vortices. ${ }^{10}$ In the present work, we study the statistical correlation between light-particle dynamics and the one of small scale vortex filaments. Previous numerical ${ }^{11}$ and experimental $^{12}$ works have assessed in great detail the spatial distribution and correlation of intense vortex filaments. Here we want to focus on different properties: their temporal evolution. Thanks to the correlation with the trajectories of light particles, we measure the vortex filament lifetime distribution.

Data come from a direct numerical simulation (DNS) of 3D fully periodic Navier-Stokes equations plus particles. Together with the Eulerian field, we integrated the Lagrangian evolution of particles by mean of a model of dilute, passively advected, suspensions of spherical particles ${ }^{13,14}$

$$
\frac{\mathrm{d} \boldsymbol{x}}{\mathrm{d} t}=\boldsymbol{v}, \quad \frac{\mathrm{d} \boldsymbol{v}}{\mathrm{d} t}=\beta \frac{\mathrm{D} \boldsymbol{u}}{\mathrm{D} t}+\frac{1}{\tau_{p}}(\boldsymbol{u}-\boldsymbol{v}) .
$$

In the above equations, $\boldsymbol{x}(t)$ and $\boldsymbol{v}(t)$ denote the particle position and velocity, respectively, $\tau_{p}=a^{2} /(3 \beta \nu)$ is the particle response time, $a$ is the particle radius, $\mathrm{St}=\tau_{p} / \tau_{\eta}$ is the Stokes number of the particle, $\tau_{\eta}$ is the dissipative time, and $\beta$ $=3 \rho_{f} /\left(\rho_{f}+2 \rho_{p}\right)$ is related to the contrast between the density of the particle, $\rho_{p}$, and that of the fluid, $\rho_{f}$. Let us stress that in the model particles are not defined in terms of their mate- rial physical properties (size and density) but in terms of their dynamical properties (response time and density contrast) and that the formula given for $\tau_{p}$ is a physical interpretation connecting the two points of view. For instance $\beta=0$ (limit of very heavy particles) with finite Stokes is then only valid assuming vanishing particle size. Moreover, light particles with large Stokes may not be compatible with the request to have a small radius. For instance, for $\mathrm{St}=0.5$ and $\beta=3$, the resulting effective size is $a=6 \eta$, where $\eta$ is the dissipative scale. For such parameters the use of point particles must be understood as a good model of transport of some kind of virtual passive markers of high vorticity. The incompressible fluid velocity $\boldsymbol{u}(\boldsymbol{x}, t)$ evolves according to the Navier-Stokes equations

$$
\mathrm{D}_{t} \boldsymbol{u} \equiv \partial_{t} \boldsymbol{u}+\boldsymbol{u} \cdot \boldsymbol{\nabla} \boldsymbol{u}=-\boldsymbol{\nabla} p+\nu \Delta \boldsymbol{u}+\boldsymbol{f},
$$

where $p$ denotes the pressure and $\boldsymbol{f}$ an external forcing keeping constant the spectral content of the two smallest wavenumber shells. We report data coming from two sets of simulations with $N^{3}=128^{3}$ and $N^{3}=512^{3}$ collocation points, corresponding to $\operatorname{Re}_{\lambda} \simeq 65$ and $\mathrm{Re}_{\lambda} \simeq 180$, respectively (see Table I for technical details on the code). We focus mainly on very light particles, in the limit of $\rho_{p} \rightarrow 0(\beta \rightarrow 3)$ and on tracers evolving with the local Eulerian velocity field $\dot{x}(t)$ $=\boldsymbol{u}[\boldsymbol{x}(t), t]$. Inertial particles are not distributed homogeneously in the volume, centrifugal forces tend to concentrate light particles inside strong elliptical regions (with high vorticity - see inset of Fig. 1) and heavy particles in hyperbolic regions, typical of intense shear. One thus expects different temporal correlations between particle trajectories and the underlying topology of the carrier flow. ${ }^{19-21}$ To quantify this effect, we show in Fig. 1 the conditional probability density of the halving times, $\tau_{1 / 2}$, of the vorticity magnitude along particle trajectories. The vorticity halving time is defined, given a time $t$, as the first time-lag after which the vorticity becomes $1 / 2$ larger or smaller than the initial value, $\tau_{1 / 2}(t)=\min [\tau \mid \omega(t+\tau) / \omega(t)=1 \pm 1 / 2]$. In order to focus only 
TABLE I. Eulerian and Lagrangian parameters. $N$ : number of grid points per spatial direction; $\operatorname{Re}_{\lambda}$ : Taylor scale Reynolds number; $\eta$ : Kolmogorov dissipative scale; $d x$ : grid spacing; $\tau_{\eta}=\sqrt{\varepsilon / \nu}$ : Kolmogorov dissipative time scale, $\varepsilon$ : energy dissipation; $\nu$ : kinematic viscosity; and $d t$ : temporal discretization. Total number of particle per Stokes value is $7.5 \times 10^{6}$ for run A and $2.5 \times 10^{5}$ for run B. The viscosity is chosen to have a Kolmogorov length scale $\eta \approx d x$. This conservative choice yields Reynolds numbers smaller than other DNS at comparable resolution (see, for example, Refs. 15 and 16). It ensures a good resolution of the small-scale velocity dynamics. We use a fully dealiased pseudospectral algorithm with second order Adams-Bashforth time-stepping (for details see Ref. 17). Particle dynamics is evolved with a time step from 10 to 1000 times smaller than the Stokes time. Velocity field at the particle position is obtained by trilinear interpolation. The numerical simulation was validated with different temporal integration scheme, different particles interpolation and different large scale forcing (Ref. 18). Some raw data are freely available from http://cfd.cineca.it.

\begin{tabular}{ccrcccccc}
\hline \hline & $N$ & $\operatorname{Re}_{\lambda}$ & $\eta$ & $d x$ & $\varepsilon$ & $\nu$ & $\tau_{\eta}$ & $d t$ \\
\hline Run A & 512 & 185 & $1 \times 10^{-2}$ & $1.2 \times 10^{-2}$ & 0.94 & $2 \times 10^{-3}$ & $4.7 \times 10^{-2}$ & $4 \times 10^{-4}$ \\
Run B & 128 & 65 & $3.4 \times 10^{-2}$ & $4.8 \times 10^{-2}$ & 0.85 & $1 \times 10^{-2}$ & $1.1 \times 10^{-1}$ & $1 \times 10^{-3}$ \\
\hline \hline
\end{tabular}

on intense turbulent events, we condition the probability density functions (PDFs) such that the vorticity magnitude at the reference time $t$ is greater than a given threshold. For the case in Fig. 1, we chose it to be $5 \omega_{\text {rms. }}$. As one can see from Fig. 1, for a given Stokes, St $\sim 0.5$, at changing the density contrast, $\beta$, one moves toward higher and higher probability to observe long halving times, i.e., light particles tend to live in regions of high and stable vorticity. In particular, particles with $\beta=1.5$ and $\beta=3$ have higher chances to get intense halving time events at comparable total statistics, a signature of the fact that light particles tend to remain trapped inside vortex filaments more than neutral or heavy particles do. The correlation between light particle position and vorticity distribution is also supported by the increased probability of light particle to fall in the region with rotation-dominated rather then dissipation-dominated flow topology as shown in Ref. 22.

In order to investigate this point more quantitatively, we extend here the stability analysis performed in Ref. 23 for the dynamics of heavy particles advected by the velocity field of a (steady) Burgers vortex ${ }^{24}$ to the case of particles with a

$$
\left\{\begin{array}{l}
\dot{x}=v_{x}, \\
\dot{y}=v_{y}, \\
\dot{v}_{x}=\left[\beta\left(A^{2}-\frac{1}{16 \pi^{2}}\right)-\frac{A}{\mathrm{St}}\right] x+\left(\frac{\beta A}{2 \pi}-\frac{1}{4 \pi \mathrm{St}}\right) y-\frac{1}{\mathrm{St}} v_{x}, \\
\dot{v}_{y}=-\left(\frac{\beta A}{2 \pi}-\frac{1}{4 \pi \mathrm{St}}\right) x+\left[\beta\left(A^{2}-\frac{1}{16 \pi^{2}}\right)-\frac{A}{\mathrm{St}}\right] y-\frac{1}{\mathrm{St}} v_{y} .
\end{array}\right.
$$

Correspondingly, the condition for the center of the vortex to be stable, in terms of the parameters, finally reads

$$
\mathrm{St}[1-2(\beta \mathrm{St}) A]^{2}<16 \pi^{2} A-(\beta \mathrm{St})\left(16 \pi^{2} A^{2}-1\right) .
$$

Moreover, since $A \sim \operatorname{Re}_{\lambda}^{-1 / 2}, 23$ we expect $A \ll 1$, hence the expression (5) can be highly simplified to generic density ratio $\beta$. The axial, radial and angular components of the model fluid velocity field associate to a Burgers vortex are given (in nondimensional form) by

$$
u_{z}=2 A z ; \quad u_{r}=-A r ; \quad u_{\theta}=\frac{1}{2 \pi r}\left[1-e^{-r^{2} / 2}\right]
$$

where $A$ is the strain parameter and $r$ is the radial distance from the vortex center, adimensionalized by the so-called vortex core size. As in Ref. 23, we will limit ourselves to consider the two-dimensional dynamics in the $(r, \theta)$ plane, where the velocity field reads (in Cartesian coordinates)

$$
\begin{aligned}
& u_{x}=-A x-\frac{y}{2 \pi r^{2}}\left[1-e^{\left(-r^{2} / 2\right)}\right], \\
& u_{y}=-A y+\frac{x}{2 \pi r^{2}}\left[1-e^{\left(-r^{2} / 2\right)}\right] .
\end{aligned}
$$

Linearizing the expressions (4) around the vortex center, $(x, y)=(0,0)$ and inserting them in Eq. (1), we get the following dynamical system for $\left(x, y, v_{x}, v_{y}\right)$ :

$$
(\beta-1)+o\left(\operatorname{Re}_{\lambda}^{-1 / 2}\right)>0 .
$$

This implies that heavy particles $(\beta<1)$, are expelled from the vortex core, while light particles $(\beta>1)$ are attracted inside it.

Stable vortex structures, chaotically advected by turbulent flows, should therefore play the role of attractive sinks in the dynamics. The larger their lifetime, the more nonhomo- 


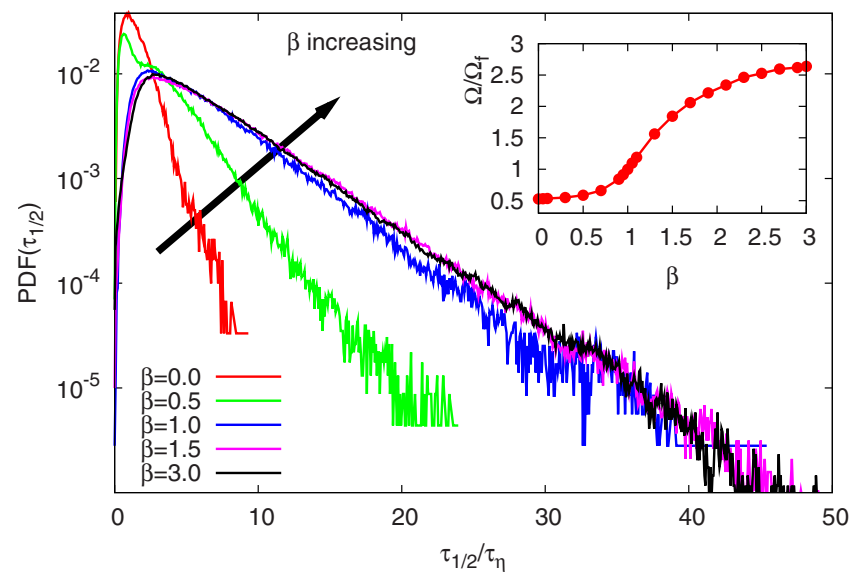

FIG. 1. (Color online) Probability density functions (PDFs) of halving times for various density ratios, $\beta$, at fixed $\mathrm{St} \sim 0.5$, conditioned on the initial value of the vorticity magnitude. As $\beta$ increases the PDFs show larger and larger tails, suggesting that lighter particles are probing regions of high and stable vorticity - the functional shape of the PDFs does not change significantly for higher values of the threshold. Inset: mean squared vorticity measured on the particle position at changing $\beta$, normalized with the $\beta=1$ case. A clear indication of preferential concentration on high vorticity region is detected for light particles with $\beta>1$.

geneous bubble distribution will develop. To quantify better the clustering properties of light particles, we focus on the statistics of the largest Lyapunov exponent, characterizing the separation rate of pairs of particles. It is known that clustering and inertia may affect the whole distribution of Lyapunov exponents. One would expect that inertia reduces the tendency for particle pairs to separate. Indeed, a weak tendency toward a reduction of the Lyapunov exponent at increasing the Stokes number has been reported for heavy particles ${ }^{25}$ similar results have also been obtained in random flows. ${ }^{26}$ Even more informative is the study of the full probability distribution of the largest finite-time Lyapunov exponent (FTLE), $\gamma_{1}(T)$ defined as

$$
\gamma_{1}(T)=\frac{1}{T} \log [R(T, t) / R(0, t)],
$$

where $R(T, t)$ is the separation of two particles at time $t+T$ starting from a separation $R(0, t)$ at time $t$. For large times, the distribution of FTLE is expected to follow a largedeviation form: $p_{T}\left(\gamma_{1}\right) \propto \exp \left[-T S\left(\gamma_{1}\right)\right]$, where the Cramér function, $S\left(\gamma_{1}\right)$ is a non-negative convex function vanishing at the value of the largest Lyapunov exponent, i.e., for $\gamma_{1}$ $=\lambda_{1}$. In Fig. 2, we report the Cramér function at $T=190 \tau_{\eta}$ for the case of light particles $\beta=3$, St $\sim 1.2$ compared with the one obtained for tracers. Two remarkable effects are visible. First, the minimum for the case of light particles is achieved for a value much smaller than the one for the tracers, precisely $\gamma_{1} \tau_{\eta} \sim 0.04$ for bubbles and $\gamma_{1} \tau_{\eta} \sim 0.14$ for tracers. Second, light particles show a remarkably high probability to have pairs that do not separate at all, even for long time lapses, i.e., there are many events in the left tail of the Cramér function which have a negative FTLE. The global average properties, however, remain chaotic (the value of $\gamma_{1}$ at which the Cramér function attains its minimum is still positive), i.e., with probability one all couples separate at

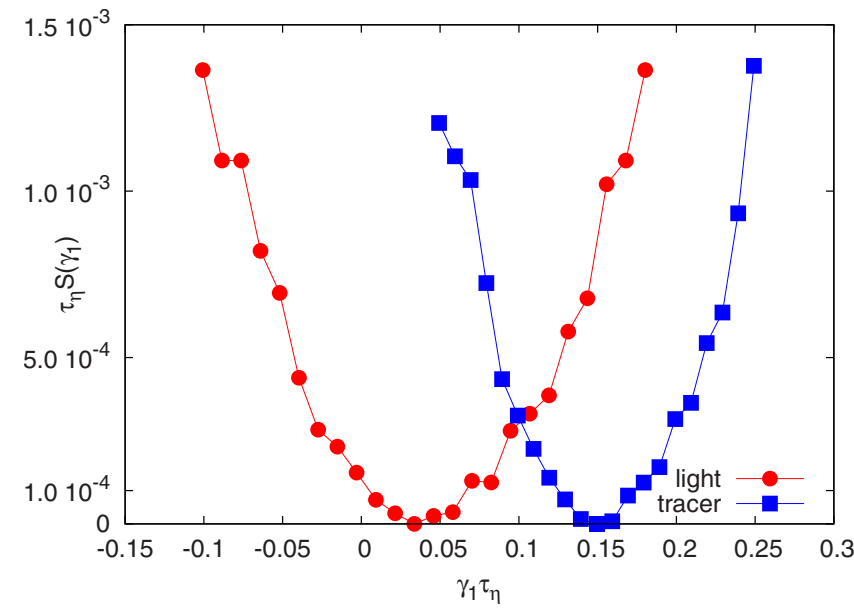

FIG. 2. (Color online) Cramér entropy for light particles and tracers. The minimum (which identifies the maximum Lyapunov exponent, $\lambda_{1}$ ) for light particles is attained for lower values. The entropy for light particles shows larger tails, thus suggesting a higher degree of intermittency. For the case of light particles, there is a non-negligible fraction of negative events, showing the existence of particle pairs that do not separate at all (absent in the tracers case).

long times. Strong clustering is also confirmed by measurements of the Kaplan-Yorke dimension, whose value, for the family of light particles shown in Fig. 2, has been found to be $D_{K Y}=1.3 \pm 0.3 ;^{27}$ thus indicating that the attractor is a quasi-one-dimensional manifold, as it would be expected for filamentary structures. The value for the fractal dimension of intense vorticity regions is consistent with results obtained with a completely different Eulerian method. ${ }^{11}$ The observation that such strong clustering at small scales is correlated with the topology of flow structures at the same scales suggests the possibility to use light particles to study statistical properties of vortex filaments in turbulence.

The identification of small scale vortex filaments is an extremely daunting task. An analysis of the Eulerian fields would require the detection of isosurfaces of vorticity (larger than some prescribed threshold): a problematic method, if not for the larger and most intense structures. ${ }^{11}$ Furthermore to analyze the temporal evolution of vortex filaments one needs to track three-dimensional (3D) structures in space and in time, by repeating the same analysis at each Eulerian time step. Our original proposal here is to use multiparticle correlations to extraordinary enhance the signal-to-noise ratio associated to the identification of vortex filaments. In Fig. 3, we show the trajectories of several light particles which are attracted into a vortex filament and that then separate again once the vortex filament disappears. Only the favorable pressure gradient, which tends to concentrate bubbles in the vortex core, permits such a strong clustering. Our goal is to identify an observable which is very sensitive to the presence of small scale vortex filaments and capable to define a birth and death time for each structure. We take a snapshot of light particles distributions at a time roughly in the middle of our numerical simulation. We divide our simulation volume into small cubes, of linear size about four times the Kolmogorov length, $\eta$, and we look for those volumes with larger particle counts. The particles residing in a volume will form what we 


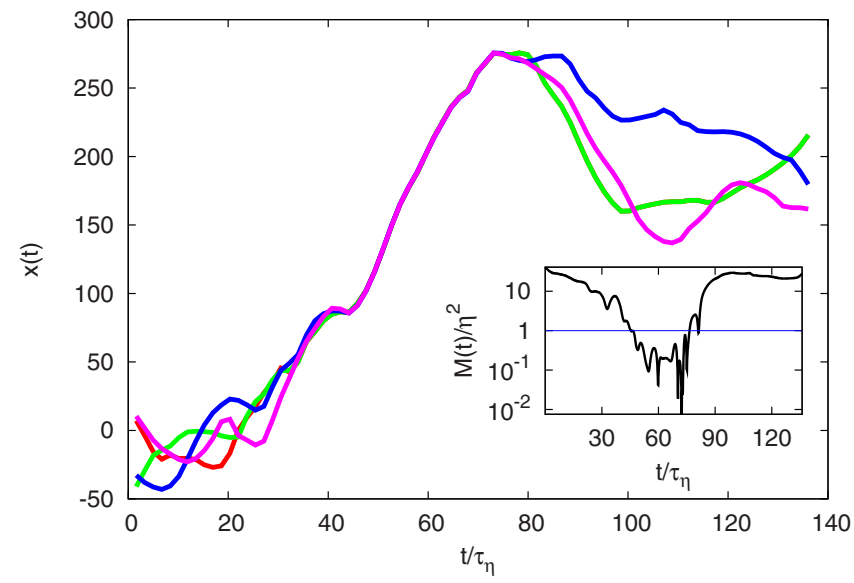

FIG. 3. (Color online) Evolution of a bunch of light particle trajectories entering in a small scale vortex filament and then separating again. Inset: behavior of the moment of inertia of the particle cloud. At entering (exiting) from the vortex filament an abrupt notice the log scale decrease (increase) of the signal allows to sharply define the lifetime of the vortex filament. For the sake of clearness we plot only four particle trajectories out of hundreds.

call a bunch. We then consider the full trajectory of the particles within each bunch. We identify $M$ bunches with the $i$ th bunch formed by $N_{i}$ particles, then we define its center of mass as $\boldsymbol{x}_{\mathrm{cm}}^{i}(t)=\left(1 / N_{i}\right) \sum_{j=1}^{N_{i}} \boldsymbol{x}_{j}(t)$ and its moment of inertia as

$$
M_{i}(t)=\frac{1}{N_{i j=1}} \sum_{i}^{N_{i}}\left[\boldsymbol{x}_{j}(t)-\boldsymbol{x}_{\mathrm{cm}}^{i}(t)\right]^{2} .
$$

The physical interpretation is clear, the smaller the moment of inertia of the bunch, the closer the particles. The important observation here is that this quantity is very sensitive to vortex filaments and displays an extraordinarily high signal/ noise ratio (see inset of Fig. 3). To understand this point one has to consider the fact that we find easily hundreds of particles at distances smaller than $0.1 \eta$, while for a uniform distribution one would expect to find $1.4 \times 10^{-3}$ particles in a $\eta^{3}$ volume. Therefore, the probability to find a finite number of particles (even if just a few, say 3 or 4 ) inside a small volume, $\eta^{3}$, is so small that when this happens it is almost surely associated to the presence of confining forces keeping the particles close by (the probability can be quickly estimated by means of a Poissonian distribution).

Another remarkable feature that makes the moment of inertia an extremely sensitive and hence useful quantity to identify vortex filaments is the rapidity with which light particles in the neighborhood of a forming vortex filament are attracted into it. In the inset of Fig. 3, one can indeed see that particles initially separated move closer, remain very close to each other for some time, and then separate again. The collapsing dynamics is quite robust, as shown also by the following experiment. In order to better understand the dynamic of light particles close to a vortex filament, we investigate what happens around a Burgers vortex. We integrate numerically Eq. (1) using the velocity field given by Eq. (4). Given the trajectory of particles, we compute the time evolution of the moment of inertia of a cloud (bunch) of light particles. In Fig. 4, we show the time evolution for $M(t)$ versus $t$ for a cloud of particles falling into a Burgers vortex, together with

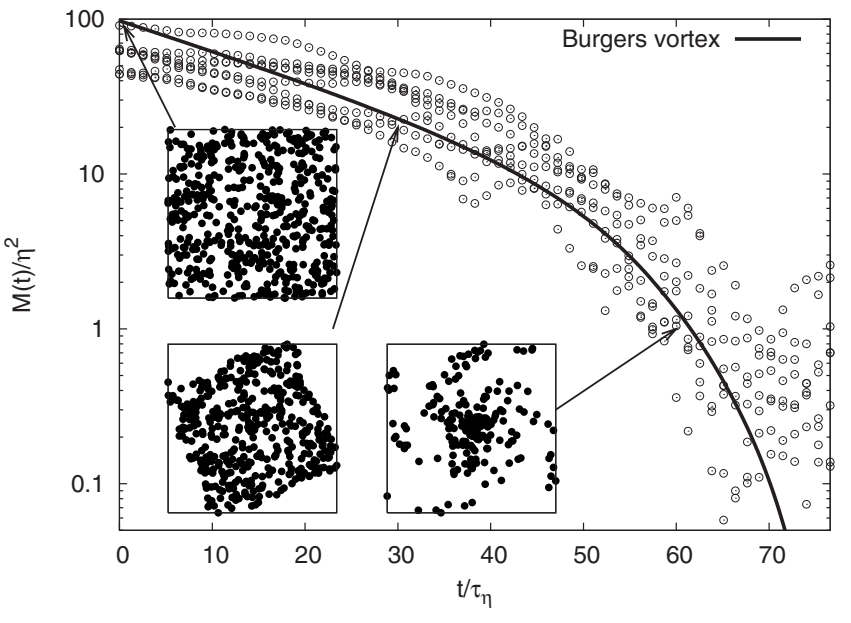

FIG. 4. Moment of inertia of bunches of light particles from the NavierStokes DNS (empty circles), compared with the value computed with the velocity field from the Burgers vortex (solid line). The three insets show the cloud of particles evolving around the Burgers vortex and falling inside it at three different instants of time, indicated with arrows along the time evolution of $M(t)$. Initial particle distribution is taken uniform in a square centered on the vortex core. Notice the formation of the typical spiral structure induced by the collapse.

the ones computed from bunches of trajectories evolved in the real DNS of Navier-Stokes equations; we find the same behavior in the trapping process for the two cases, within some fluctuations among the DNS signals, being, perhaps, attributable to a residual motion in the vertical coordinate (absent in the synthetic vortex case).

Going back to the light particle dynamics in the DNS, by analyzing the statistics of all bunches, we can make a histogram of vortex filament lifetimes, allowing-for the first time- to quantitatively assess the statistical properties of these extreme events. In Fig. 5, we show the PDF of vortex filament lifetimes for $\operatorname{Re}_{\lambda}=180$ and 65 which happens to be

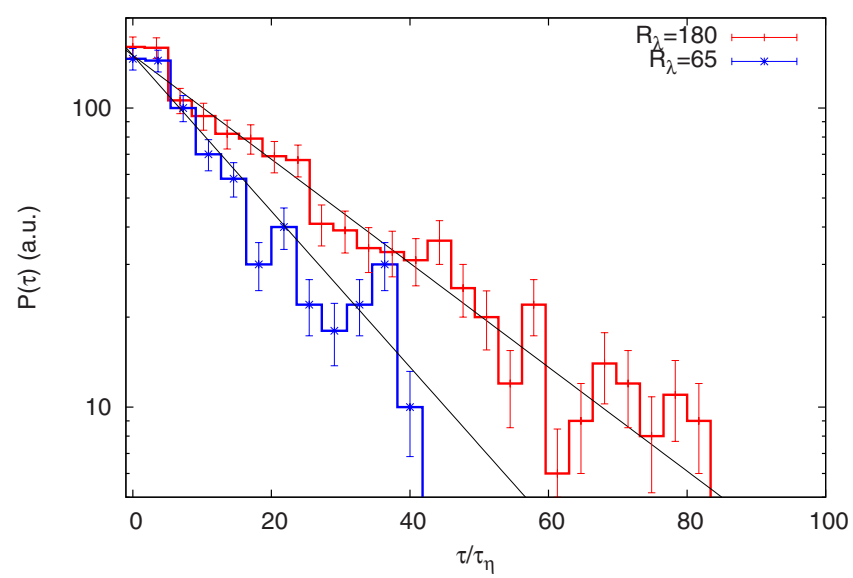

FIG. 5. (Color online) PDFs of vortex filaments lifetimes at two different Reynolds number, $R_{\lambda}=65,180$ : in both cases the PDF is fairly fitted by an exponential (with a decay factor, for the highest $R_{\lambda}$, of around $25 \tau_{\eta}$ ). In the far tail there are events whose lifetime is of the order of the integral time. Error bars reflect the effect of changing the threshold used to determine the vortex filament lifetime. 
an exponential with a decay rate which can be estimated of the order of $25 \tau_{\eta}$ and $17 \tau_{\eta}$, respectively. As already noticed in Ref. 7, we do observe events as long as the integral time $T_{L}$, estimated to be in the order of $50 \tau_{\eta}$, for the largest Reynolds. The extreme sharpness (rapidity) with which the moment of inertia drops and then raises again allows to define the lifetime of the vortex filament in a robust way. Due to this important fact, the estimate of the lifetime is not much sensitive to the chosen threshold (in Fig. 3 the threshold has been set equal to 1 , changing the value of the threshold is accounted into the error bars of Fig. 5).

One of the most intriguing features of fluid dynamics turbulence is the presence of long living coherent structures at small scales. The quantification of the statistical properties of these Eulerian structures has always proved to be one of the most difficult statistical analysis in turbulence due to the extremely low signal to noise ratio and to the need of following the evolution of structures for as long as a large scale eddy turnover time. Here, we have showed that vortex filaments act as strong sink in the dynamics of light particles, making the dynamics of particle pairs, or particle bunches, strongly confined on quasi-one-dimensional structures. Moreover, a comparison between the evolution into a Burgers vortex and light particles evolving in a fully resolved DNS showed remarkable agreement.

We quantified the probability distribution function of particle pair separation, by studying the Cramér function and the vortex lifetimes; both measurements show events up to the integral time scale, $T_{L}$. A very important open issue would be to quantify the Reynolds number dependency of such effects. Unfortunately, it is not possible, at the moment, to reach much higher Reynolds numbers in numerics. A possible way out could be to perform careful experiments capable to track clouds of bubbles in turbulence for a relatively long period of time, at high Reynolds numbers.

We thank the DEISA Consortium (cofunded by the EU, FP6 Project No. 508830) for support within the DEISA Extreme Computing Initiative (www.deisa.org). We thank CASPUR (Italy) and CINECA (Italy) for the computational resources. Data from this study are publicly available in unprocessed raw format from the iCFDdatabase (http://cfd.cineca.it).

${ }^{1}$ P. G. Saffman, Vortex Dynamics (Cambridge University Press, Cambridge, England, 1992).

${ }^{2}$ H. K. Moffatt, S. Kida, and K. Ohkitani, "Stretched vortices-the sinews of turbulence; large-Reynolds-number asymptotics," J. Fluid Mech. 259, 241 (1994)

${ }^{3}$ S. Chen, G. L. Eyink, M. Wan, and Z. Xiao, "Is the Kelvin theorem valid for high Reynolds number turbulence?" Phys. Rev. Lett. 97, 144505 (2006).
${ }^{4}$ S. Kida and M. Takaoka, "Vortex reconnection," Annu. Rev. Fluid Mech. 26, 169 (1994).

${ }^{5} \mathrm{~F}$. Toschi and E. Bodenschatz, "Lagrangian properties of particles in turbulence," Annu. Rev. Fluid Mech. 41, 375 (2009).

${ }^{6}$ J. K. Eaton and J. R. Fessler, "Preferential concentrations of particles by turbulence," Int. J. Multiphase Flow 20, 169 (1994).

${ }^{7}$ S. Douady, Y. Couder, and M.-E. Brachet, "Direct observation of the intermittency of intense vorticity filaments in turbulence," Phys. Rev. Lett. 67, 983 (1991).

${ }^{8}$ D. Bonn, Y. Couder, P. H. J. van Dam, and S. Douady, "From small scales to large scales in three-dimensional turbulence: The effect of diluted polymers," Phys. Rev. E 47, R28 (1993).

${ }^{9}$ A. La Porta, G. A. Voth, F. Moisy, and E. Bodenschatz, "Using cavitation to measure statistics of low-pressure events in large-Reynolds-number turbulence," Phys. Fluids 12, 1485 (2000).

${ }^{10}$ G. P. Bewley, D. P. Lathrop, and K. R. Sreenivasan, "Superfluid helium: Visualization of quantized vortices," Nature (London) 441, 588 (2006).

${ }^{11}$ F. Moisy and J. Jiménez, "Geometry and clustering of intense structures in isotropic turbulence," J. Fluid Mech. 513, 111 (2004).

${ }^{12}$ H. Mouri, A. Hori, and Y. Kawashima, "Laboratory experiments for intense vortical structures in turbulence velocity fields," Phys. Fluids 19, 055101 (2007).

${ }^{13}$ M. R. Maxey and J. J. Riley, "Equation of motion of a small rigid sphere in a nonuniform flow," Phys. Fluids 26, 883 (1983).

${ }^{14}$ T. Auton, J. Hunt, and M. Prud'homme, "The force exerted on a body in inviscid unsteady nonuniform rotational flow," J. Fluid Mech. 197, 241 (1988).

${ }^{15}$ T. Ishihara, T. Gotoh, and Y. Kaneda, "Study of high-Reynolds turbulence by direct numerical simulation," Annu. Rev. Fluid Mech. 41, 165 (2009).

${ }^{16}$ P. K. Yeung, S. B. Pope, and B. L. Sawford, "Reynolds number dependence of Lagrangian statistics in large numerical simulations of isotropic turbulence," J. Turbul. 7, 58 (2006).

${ }^{17}$ J. Bec, L. Biferale, G. Boffetta, A. Celani, M. Cencini, A. Lanotte, S. Musacchio, and F. Toschi, "Acceleration statistics of heavy particles in turbulence,” J. Fluid Mech. 550, 349 (2006).

${ }^{18}$ E. Calzavarini, R. Volk, M. Bourgoin, E. Lévèque, J.-F. Pinton, and F. Toschi, "Effects of Faxén forces on acceleration statistics of material particles in turbulent flow," in Advances in Turbulence XII, edited by B. Eckhardt (Springer, New York, 2009), p. 11.

${ }^{19}$ P. K. Yeung, S. B. Pope, E. A. Kurth, and A. G. Lamorgese, "Lagrangian conditional statistics, acceleration and local relative motion in numerically simulated isotropic turbulence," J. Fluid Mech. 582, 399 (2007).

${ }^{20}$ B. Lüthi, A. Tsinober, and W. Kinzelbach, "Lagrangian measurement of vorticity dynamics in turbulent flow," J. Fluid Mech. 528, 87 (2005).

${ }^{21}$ M. Guala, B. Lüthi, A. Liberzon, A. Tsinober, and W. Kinzelbach, "On the evolution of material lines and vorticity in homogeneous turbulence," J. Fluid Mech. 533, 339 (2005).

${ }^{22}$ R. Benzi, L. Biferale, E. Calzavarini, D. Lohse, and F. Toschi, "Velocity gradients statistics along particle trajectories in turbulent flows: The refined similarity hypothesis in the Lagrangian frame," Phys. Rev. E 80, 066318 (2009).

${ }^{23}$ B. Marcu, E. Meiburg, and P. K. Newton, "Dynamics of heavy particles in a Burgers vortex," Phys. Fluids 7, 400 (1995).

${ }^{24}$ J. M. Burgers, "A mathematical model illustrating the theory of turbulence," Adv. Appl. Mech. 1, 171 (1948).

${ }^{25}$ J. Bec, L. Biferale, G. Boffetta, M. Cencini, S. Musacchio, and F. Toschi, "Lyapunov exponents of heavy particles in turbulence," Phys. Fluids 18, 091702 (2006).

${ }^{26}$ K. Duncan, B. Mehlig, S. Östlund, and M. Wilkinson, "Clustering by mixing flows," Phys. Rev. Lett. 95, 240602 (2005).

${ }^{27}$ E. Calzavarini, M. Kerscher, D. Lohse, and F. Toschi, "Dimensionality and morphology of particle and bubble clusters in turbulent flow," J. Fluid Mech. 607, 13 (2008). 